\title{
Intraosseous Squamous Cell Carcinoma Arising from Chronic Osteomyelitis: A Case Report and Review of the Literature
}

\author{
Yu-De Su and Po-Yin Shen* \\ Department of Orthopaedics, Kaohsiung Medical University Hospital, Taiwan \\ *Corresponding author: Po-Yin Shen, Department of Orthopaedics, Kaohsiung Medical University Hospital, Taiwan
}

\section{ARTICLE INFO}

Received: 彗 April 02, 2019

Published: May 13, 2019

Citation: Yu-De Su, Po-Yin Shen. Acute Intraosseous Squamous Cell Carcinoma Arising from Chronic Osteomyelitis: A Case Report and Review of the Literature. Biomed J Sci \& Tech Res 17(5)2019. BJSTR. MS.ID.003077.

Keywords: Osteomyelitis; Squamous Cell Carcinoma; Malignant Tumors

Abbreviations: MRI: Magnetic Resonance Imaging, OPD: Out-Patient Department
ABSTRACT

Background: Chronic osteomyelitis is common in Orthopedic clinical practice, but malignant transformation is a rare and late complication. We report one patient with left thigh squamous cell carcinoma arising from chronic osteomyelitis.

Case Report: A 68-year-old patient sustained trauma to the left lower limb with femur and tibia fracture 30 years ago. Chronic osteomyelitis developed after osteosynthetic procedure. Several debridement and sequestrectomy were done but with little help. Sinus tract discharge with left thigh palpable painful mass was noted about 7 years ago. After repeated surgical debridement, sequestrectomy, and serial MRI exams, the result showed malignant transformation.

Discussion: Squamous cell carcinoma is the most common type of malignant tumor resulting from chronic osteomyelitis. It usually involves tibia, femur and foot. The mean age was 54 60-year-old, and the interval between onset of osteomyelitis and diagnosis of malignancy was 20 40 years. Symptoms includes local swelling and sinus tract discharge with unpleasant odor. MRI is the useful tool to differentiate the squamous cell carcinoma from other etiologies. Amputation proximal to the neoplasm remain the gold standard for treatment while wide resection is indicated only in selected patients.

Conclusion: Malignant transform from chronic osteomyelitis is a rare lesion in the lower extremities. Surgeon should be aware of the risk of malignant degeneration in patient with osteomyelitis and chronic wound. Early diagnosis and definitive treatment are fundamental for the prognosis and result.

\section{Introduction}

Chronic osteomyelitis is common in daily practice, but malignant transformation was rare. Hawkins was the first to document the process of squamous cell carcinoma arising in chronic osteomyelitis [1]. The physiopathological mechanism remains unclear. The increased in fistulous discharge, erythematous change, growth of the ulcer may be the red flag signs for malignant transformation. The most definitive surgical treatment in these situations is the limb amputation. We report one case with squamous cell carcinoma arising from a chronic osteomyelitis and discuss through literature review.

\section{Case Report}

This 68-year-oldmale sustained traumato the leftlegin1990 with left femoral and tibia shaft fracture. He undergone osteosynthesis surgical intervention and received antibiotic treatment at that time. Unfortunately, left distal femur chronic osteomyelitis with sinus tract discharge developed (Figure 1). He received the surgery to remove implant, sequestrectomy, debridement, and local skin flap for many times. With newly developed palpable mass at anterolateral aspect of thigh and recurrent sinus tract discharge 7 years ago, he came to the out-patient department (OPD) for help. Physical exam revealed local tenderness, swelling, erythematous change, with small palpable mass over anterior thigh. Magnetic resonance imaging (MRI) (Figure 2) revealed that the abscess formation surrounded the distal femur and adjacent tissue, with infiltration of left thigh muscle. Repeat debridement and soft tissue tumor excision were performed. The histopathology report that left thigh mass was the soft tissue tumor with squamous epithelial 
hyperplasia with mild atypia. Ten months later, he noticed that the mass regrew and continued to increase in size, causing skin tenting and wound poor healing.

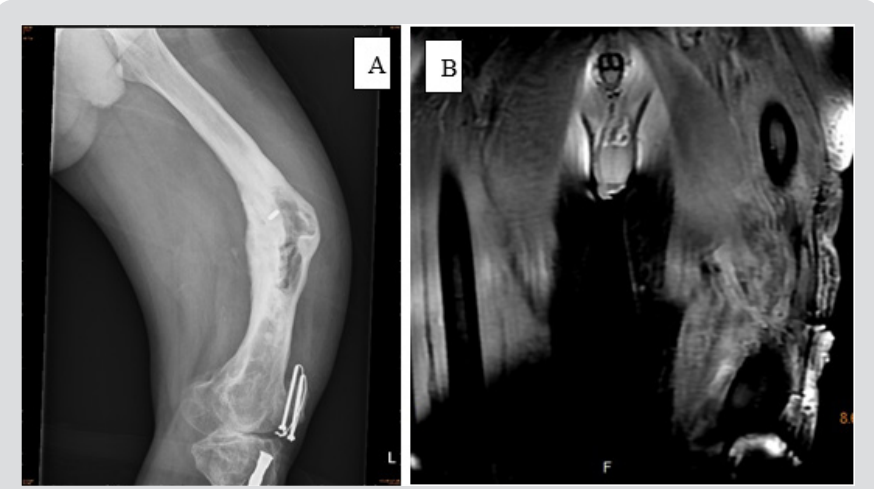

Figure 1: Anteroposterior X-ray

A. Presenting initial chronic osteomyelitis with femur varus deformity and lateral cortex erosion. MRI

B. Presenting left femur osteomyelitis with infectious status of muscle and abscess formation.

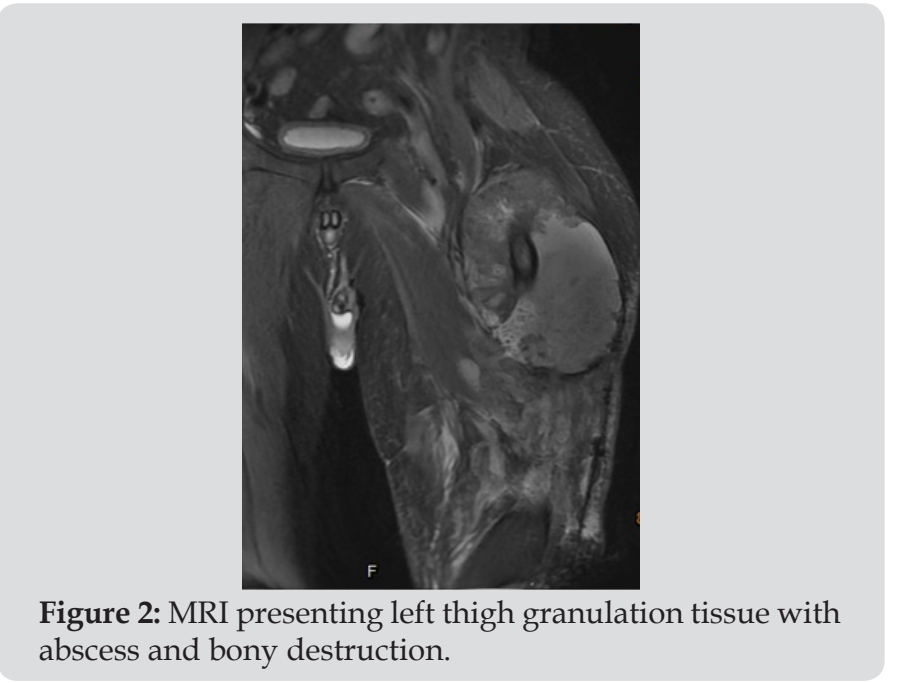

The patient visited our OPD again and was thus referred to the Orthopedic oncology surgeon for evaluation. Clinical exam showed a $6 \times 4 \mathrm{~cm}$, firm, irregular, immobile mass extending from the anterior to the lateral aspect of left thigh. There was no numbness or weakness or sensory defect in the affected lower limb. Radiography revealed ill-defined osteolytic lesions with sclerotic changes in left distal femur. MRI showed enlargement of granulation tissue and hematoma with adjacent bone destruction. We repeated the surgery of tumor excision. The final pathology result was identical with previous finding (squamous epithelial hyperplasia with mild atypia). After discharge for four months, sudden onset of left thigh pain was noted at distal part after standing up at home. At emergency department, radiography revealed left distal femur osteolytic lesion with impending pathological fracture (Figure 3A). Angiography was arranged for further evaluation, which revealed a bulky hypervascular soft tissue with malignancy characteristic in the left upper thigh. During the operation, massive yellow-brownish pus with lymphangio-like lobulated mass were found.
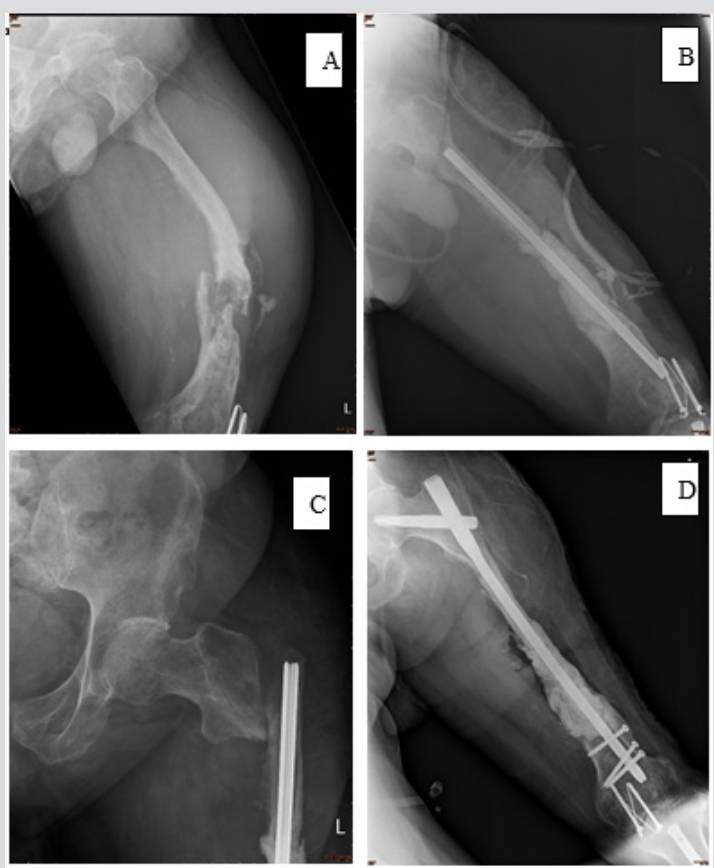

Figure 3: Anteroposterior

A. X-ray revealed distal femur pathological fracture with varus deformity,

B. Kuntsher nail for internal fixation with vaconcomysin-coated cement,

C. Left femur subtrochanteric pathological fracture (one week after Kuntsher nail fixation),

D. Remove Kuntsher nail and use long cephalomedullary nail.

In order to correct distal femur varas deformity, we performed valgus osteotomy and fixation with Kuntsher nail $(10 \mathrm{mmx} 300 \mathrm{~mm})$, and Vancomycin-coated cement spacer at fracture site (Figure 3B). The final histopathology revealed squamous cell carcinoma. However, he complained left hip pain after bedside ambulation one week later. Radiography showed left hip subtrochanteric pathological fracture, so we exchange the Kuntsher nail with Cephalomedullary nail for fixation (Figure 3C \& 3D). On the outpatient department follow-up, poor skin healing with fistula purulent discharge was still noted. Repeated MRI revealed tumor progression with involvement of gluteal muscle, anterior compartment of thigh, and left inguinal area, and the whole-body bone scan (Figure 4) had increased uptake over left inguinal area. Several debridement and tumor wide excision provided little help, so he underwent above-knee amputation. Five months later, left anterior palpable mass with anterior thigh pain and skin tenting was noted again. Repeat MRI (Figure 5) revealed recurrence of malignant tumor, lymphadenopathy in the left groin region, and peri-acetabular bone metastasis. Thus, we preceded with left hemipelvectomy (Figure 6) and skin coverage with local fasciocutaneous flap. After the surgery, the patient received adjuvant chemotherapy therapy. The pain resolved, and there was no marked local recurrence over a period of 2 years. 


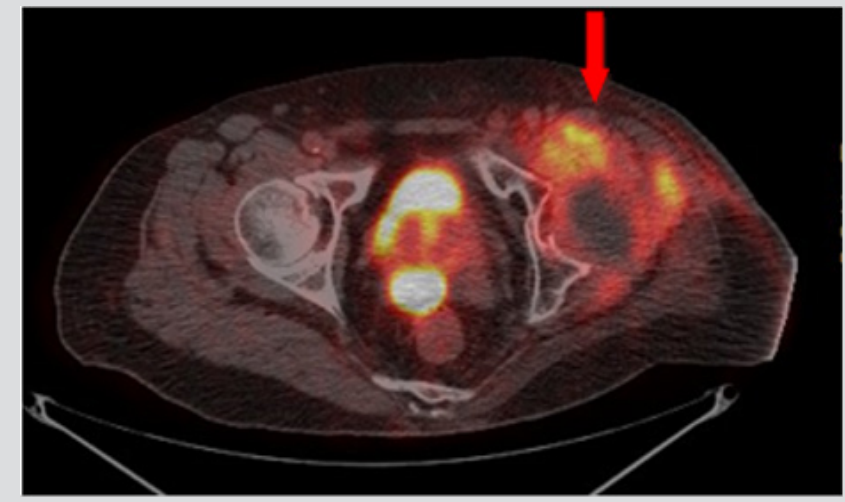

Figure 4: Whole body bone scan presenting increased radioactivity over anterior aspect of left inguinal area.

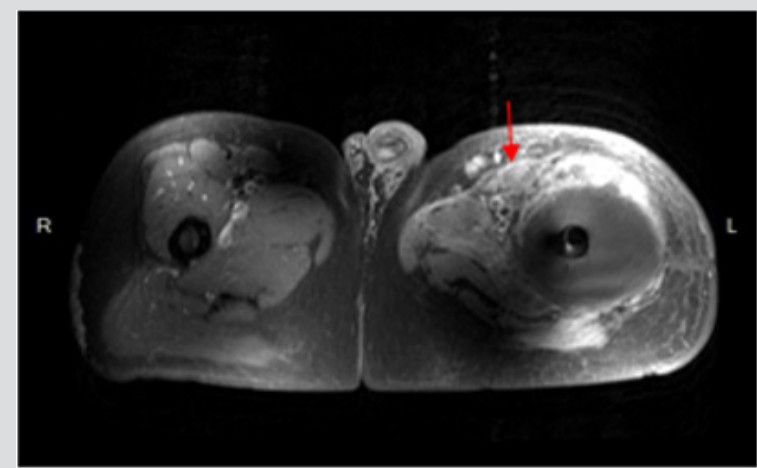

Figure 5: MRI revealed some soft tissue component (Arrow) anterior to the abscess formation, and lymphadenopathy in the left groin region.

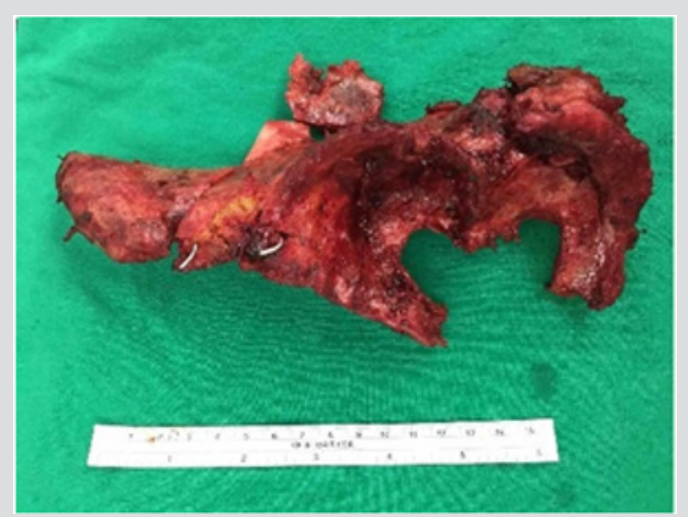

Figure 6: Photography of left pelvic bone (post left hemipelvectomy).

\section{Discussion}

Squamous cell carcinoma is the most common type of malignant tumor resulting from chronic osteomyelitis [1]. The most common site of involvement is tibia, followed by femur and foot [2, 3]. The average age of patients with chronic osteomyelitis related squamous cell carcinoma was 54-60-year-old [3], with male predominance $[1,3]$. The interval between the onset of osteomyelitis and the diagnosis of malignant transformation was $20 \sim 40$ years [2,4], with minimum latency period of 20 years or more [3]. In our case, the patient was 68 years of age and the interval was 22 years, which almost compatible with the current report. Chronic osteomyelitis poses a risk of transformation to squamous cell carcinoma. Some author reported that over $25 \%$ of malignant tumor arises from chronic infection and inflammation, and 1.6 23\% from chronic osteomyelitis [5]. However, the exact mechanism remains unclear and is assumed multifactorial. Squamous cell carcinoma can arise from fistula tract which connects the malignant of epidermal lining.

One hypothesis clarify that inflammatory cytokines may lead to an altered expression of tumor suppression genes causing carcinogenic transformation [6], while other hypothesis clarifies that all cell types in the chronic inflammation may be susceptible to malignant change [4]. The clinical suspicion about malignant transformation including increased in mass size, ulcers, swelling, tenderness, fistula or sinus tract discharge with odor smell, and progressive disabling pain [4,5]. Plain radiography often appears bony erosion, destruction, scalloping, or even pathologic fracture in severe cases. MRI can be useful to differentiate squamous cell carcinoma from other soft tissue tumor [3] and identify adjacent lymphadenopathy. Whole body bone scan can clarify the suspicion of distant metastasis. In our case, MRI revealed lymphadenopathy in the left groin and peri-acetabular region after primary aboveknee amputation. These finding provide us more information on the decision to perform left hemipelvectomy for tumor control.

Most studies declared thatamputation is the definitive treatment for carcinomatous transformations of chronic bone infection. Amputation proximal to the lesion can decrease local recurrence and risk of metastasis, as well as quicker and safer recovery. Wide resection may be one of surgical choices in selected patients, but it's associated with high rate of local recurrence and large skin defects. Massive soft tissue, muscle, and skin defect can pose a challenge for reconstruction. The distant metastasis and lymphadenopathy had been observed in 10 20\% of patients [7], while some report rates more than $40 \%$ [6]. Yener et al. report that two patients with malignant lymphadenopathy and distant metastasis all died within one year even under re-amputation [7]. The presence of lymphatic node metastasis or distant metastasis will lower the survival rate and indicate poor prognosis. Diogo et al. [5] mentioned that hemipelvectomy may be required if invasion of lumbar-aortic lymph node. Concerning the high risk of surgery and difficulty in skin coverage, he decided against it. In our case, we performed disarticulation of the hip and lymphadenectomy with care to avoid neurovascular insult, followed by local fasciocutaneous flap for skin coverage. Early recognition and timely therapy are considerably to improve prognosis and decrease the risk of metastasis. Adjuvant chemo radiotherapy is indicated in metastatic patient [5]. In our patients, adjuvant chemotherapy with Cisplatin $(75 \mathrm{mg} / \mathrm{m} 2)$ and 5-FU $\left(1000 \mathrm{mg} / \mathrm{m}^{2}\right)$ was applied.

\section{Conclusion}

Malignant transformation in chronic osteomyelitis is a rare lesion in the lower extremity but unfavorable condition. Early 
diagnosis and definitive treatment are fundamental for the prognosis and results. Currently, amputation appears to be the gold standard for treatment.

\section{Acknowledgement}

Yu-De Su carried out the patient database and drafted the manuscript. Po-Yin Shen conceived of the study and helped to draft the manuscript.

\section{Conflict of Interest}

All authors declare that no support, financial or otherwise, has been received from any organization that may have an interest in the submitted work.

\section{References}

1. Li Q Cui H, Dong J, He Y, Zhou D, et al. (2015) Squamous cell carcinoma resulting from chronic osteomyelitis: a retrospective study of 8 case. Int J Clin Exp Pathol 8(9): 10178-10184.

ISSN: 2574-1241

DOI: 10.26717/BJSTR.2019.17.003077

Po-Yin Shen. Biomed J Sci \& Tech Res

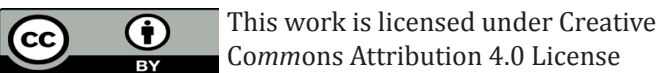

Submission Link: https://biomedres.us/submit-manuscript.php
2. Alami M, Mahfoud M, El Bardouni A, Berrada MS, El Yaacoubi M (2011) Squamous cell carcinoma arising from chronic osteomyelitis. Acta Orthop Traumatol Turc 45(3): 144-148.

3. Caruso G, Gerace E, Lorusso V, Cultrera R, Moretti L, Massari L (2016) Squamous cell carcinoma in chronic osteomyelitis: A case report and review of the literature. J Med Case Rep 10: 215.

4. Noonan KJ, Goetz DD, Marsh JL, Peterson KK (1993) Rapidly destructive squamous cell carcinoma as a complication of chronic osteomyelitis. Orthopedics 16(10): 1140-1144.

5. Moura DL, Ferreira R, Garruço A (2017) Malignant transformation in chronic osteomyelitis. Rev Bras Ortop 52(2): 141-147.

6. Panteli M, Puttaswamaiah R, Lowenberg DW, Giannoudis PV (2014) Malignant Transformation in Chronic Osteomyelitis: Recognition and Principles of Management. J Am Acad Orthop Surg 22(9): 586-594.

7. Saglik Y, Arikan M, Altay M, Yildiz Y (2001) Squamous cell carcinoma arising in chronic osteomyelitis. International Orthopedics (SCIOT) 25(6): 389-391.

$\begin{array}{ll}\text { BIOMEDICAL } & \text { Assets of Publishing with us } \\ \text { RESEARCHES } & \text { - Global archiving of articles } \\ & \text { - Immediate, unrestricted online access } \\ \end{array}$

